\title{
Deux nouvelles espèces pour la faune de France : Rhabdiop- teryx thienemanni Illies et Leuctra pasquinii Consiglio (Plecoptera)
}

\section{B. Dumont1}

Deux nouvelles espèces de Plécoptères pour la faune de France (Rhabdiopteryx thienemanni - Taeniopterygidae et Leuctra pasquinii - Leuctridae) ont été découvertes dans le Sud-Est. Leurs présences dans cette région soulignent l'affinité de cette zone avec les pays méridionaux voisins, l'Espagne et l'Ltalie, où le Taeniopterygidae espagnol et le Leuctridae italien étaient considérés jusqu'alors comme espèces endémiques.

Two additions to the French fauna : Rhabdioptery thienemani Illies and Leuctra pasquinii Consiglio (Plecoptera).

$R h$. thienemanti was previously recorded only in the Iberian Peninsula and $L$. pasquinii only in Italy. Their presence in southeastern France emphasizes the faunal affinites of this region with the neighbouring Mediterranean countries.

\section{1. - Taeniopterygidae: Rhabdiopteryx thiene- manni Illies, 1957}

\begin{abstract}
Massif des Maures. Département du Var - Ruisseau de la Verne. Altitude $300-400 \mathrm{~m}$. Affluent de la Môle. Bassin versant du Golfe de St-Tropez (voir figure). 25.1.1980: $10.6 \%$. - 17.2.1980:13 $\sigma, 12 \%, 12$ larves. -26.2 .1980 : 10,1 Q, 1 larve.

L'espèce n'était connue que de la péninsule ibérique dans un triangle Guarda (Portugal), Madrid et León (Espagne). Illies (1957) avait décrit cette nouvelle espèce avec un seul adulte mâle capturé dans la région de Madrid. Quinze ans plus tard, Zwick (1972) en décrivit l'adulte femelle ainsi que les larves des deux sexes. Berthélemy \& Gonzàlez del Tánago (1983) en mentionnent la présence dans d'autres localités espagnoles.
\end{abstract}

\footnotetext{
1. Cemagref le Tholonet, Centre National du Machinisme Agri. cole, du Gènie Rural, des Eaux et des Forêts, B.P. 99, F-13603 Aixen-Provence.
}

La comparaison avec les exemplaires portugais ne justifie pas, selon P. Zwick (com. pers.), une appellation particulière pour la population provençale. Habitat.

Les larves de $R$. thienemanni ont été rencontrées dans un cours d'eau typiquement temporaire dont l'assèchement se produit en moyenne entre le 15 juin et la fin septembre. Dans le secteur considéré l'écoulement est permanent d'octobre à mai et le cycle thermique se réalise entre $1^{\circ} \mathrm{C}$, au plus fort de l'hiver, et $27^{\circ} \mathrm{C}$ quand l'écoulement superficiel dispa. raît au début de l'été. La minéralisation des eaux est faible et la conductivité est en moyenne de $50 \mu \mathrm{S}$. Discussion.

La présence de $R$. thienemanni dans cette zone de la Provence cristalline (Gneiss) souligne à la fois l'originalité de cette région pour la France continentale et son appartenance a un grand système méridional dont seuls quelques ilots faunistiques sont connus. En effet le ruisseau de la Verne présente d'autres particularités faunistiques qui n'étaient connues jusqu'alors que des îles Canaries, de l'île de Madère et de la Corse (matériel en cours d'examen). 

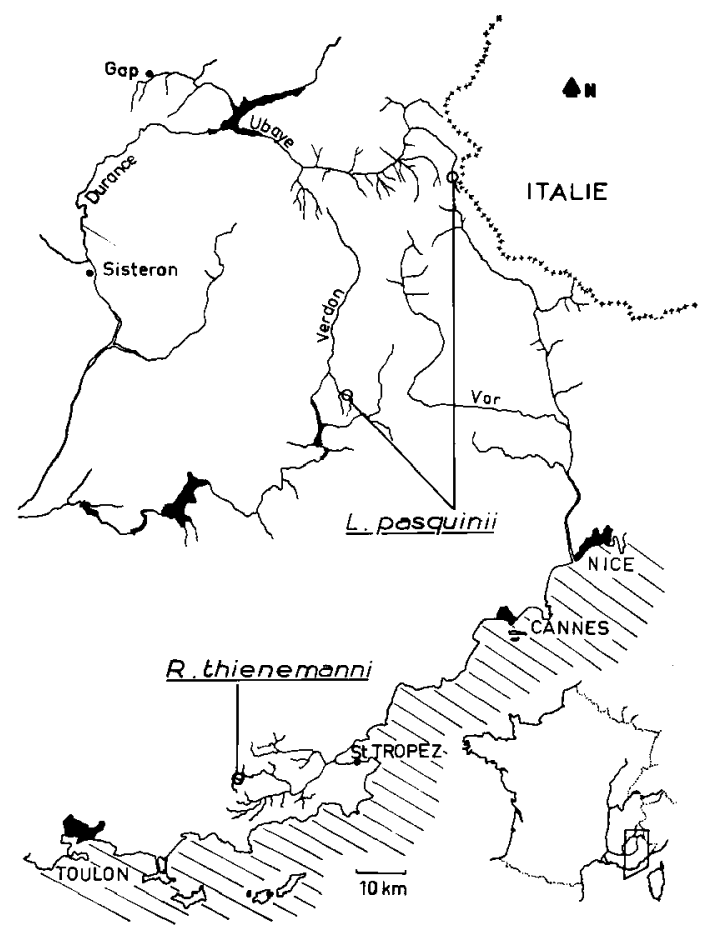

Situation géographique des stations françaises de $R$. thienemanni et $L$ pasquinii.

\section{2. - Leuctridae : Leuctra pasquinii Consiglio, 1958}

Alpes de Haute-Provence. Bassin versant du HautVerdon. Cours d'eau de l'Ivoire, affluent de rive gauche du Verdon amont de St-André-les-Alpes. Altitude $1100 \mathrm{~m}$ (voir figure) 14.9.1977:6 $\sigma, 9$ २. $-23.11 .1977: 2 \%$. $11.9 .1980: 3$ \%.

Alpes de Haute-Provence. Bassin versant de l'Ubaye. Cours d'eau l'Ubayette. Zones de sources marécageuses au bas du ravin des Eyssalps. Altitude $1970 \mathrm{~m}$. (Parc National du Mercantour). 11.10.1983:8 $\sigma, 9$ \%.

L. pasquinit était connue jusqu'alors comme une espèce italienne localisée dans la chaine montagneuse des Apennins. Consiglio (1958) la décrivit à partir d'exemplaires capturés dans les Abruzzes. Et plus récemment Ravizza et Ravizza Dematteis (1974 et 1979) mentionnent la capture de cet te espèce dans les Apennins lombards sur la rivière Staffora et dans le nord des Apennins sur la rivière Nure, affluent de rive gauche du Pô.

\section{Habitat.}

L'Ivoire coule sur un substrat calcaire (Crétacé) et la station de récolte est située à l'aval immédiat d'une zone de résurgences. L'amplitude thermique au cours d'un cycle annuel y est faible, entre $4^{\circ} \mathrm{C}$ l'hiver et $15^{\circ} \mathrm{C}$ l'été. La conductivité moyenne est de l'ordre de $280 \mu \mathrm{S}$. 
La deuxième station découverte en octobre 1983 dans la vallée de l'Ubayet te est une zone de sources limnocrènes distincte du cours d'eau principal.

\section{Discussion.}

Consiglio (1958) mentionnait que L. pasquinii vit dans des eaux fraîches $\left(5\right.$ à $\left.15^{\circ} \mathrm{C}\right)$. Les récoltes réalisées par C. Ravizza el E. Ravizza Dematteis (1974) montrent que le plus grand nombre de captures est obtenu dans les deux premiers kilomètres des cours d'eau. Ce fait est confirmé par l'implantation des deux stations françaises où $L$. pasquinii est manifestement associée à des zones de sources, en particulier dans la station de l'Ubayette où les adultes ont été capturés sur les herbes et arbustes bordant une émergence de nappe.

\section{Travaux cltés}

Berthélemy (C.) \& Gonzàlez del Tánago (M.). 1983. - Les Taenioptervgidae du bassin du Duero (Insecta : Plecoptera. Annls Limnol., 19 (1): 9-16.

Consiglio (C. ), 1958, - Contributo alla conoscenza dei Plecotteri olognati dell'lialia Centrale. Mem. Soc. ent. it., $37: 117-131$.

Illies (J.). 1957. - Beitrag zur Kenninis der Gattung Rhabdiopteryx Klap. (Plecoptera). A rch. Hydrobiol, 53 (4) : 455-464.

Ravizza (C). 1974. - Ricerche ecologica faunistiche sui Plecotteri della Val Staffora (Apennino Lombardo). Boll. Mus. civ. St. nat. Verona, 1 : 195-248.

Ravizza (C.) \& Ravizza Dernatteis (E.). 1979. - I Plecotteri del corso medio-inferiore del torrente Nure (Appennino Piacentino). Nafura, 70 (3): 193-217

Zwick (P.). 1972. - Plecoptera (Ins.) aus dem Mittelmeergebiet, vor allem aus Portugal und Spanien. Ciênc, biol. 1:7.17. 